\title{
Nueva familia de angiospermas para el registro de la flora nativa de Chile: la familia Basellaceae
}

\section{New angiosperm family for the checklist of the native Chilean flora: the family Basellaceae}

\author{
Andrés Moreira-Muñoz ${ }^{1 *} \&$ Mélica Muñoz-SchicK² \\ ${ }^{1}$ Instituto de Geografía, Pontificia Universidad Católica de Valparaíso, Av. Brasil 2241, Valparaíso, Chile. \\ ${ }^{2}$ Museo Nacional de Historia Natural, Casilla 787, Santiago, Chile. \\ *andres.moreira@pucv.cl
}

\begin{abstract}
The presence of Anredera diffusa, from the Basellaceae family, is reported for the first time for the Chilean flora. This species was found in the locality of Mancaruma, north of Socoroma town, in the Arica y Parinacota Region. This is the first record of a native species of Basellaceae in Chile.
\end{abstract}

El conocimiento de la flora de Chile ha sido construido paulatinamente a partir de los tratamientos florísticos y estadísticos de C. Gay, Rodulfoy Federico Philippi, C. Reiche, C. Muñoz Pizarro y C. Marticorena (Marticorena 1990). La estadística de Reiche (2013) incluía 141 familias, mientras Marticorena consideró 175 familias de plantas vasculares (Marticorena 1990). Debido a los cambios sistemáticos más recientes, especialmente a partir de datos moleculares, muchas familias han sido fragmentadas y otras han sido unificadas. La revisión de Moreira-Muñoz (2011), considera la presencia de 171 familias de plantas vasculares nativas en Chile. Aunque ha habido cambios nomenclaturales (e.g. Atherospermataceae, Berberidopsidaceae, Calceolariaceae, Cardiopteridaceae, Francoaceae, Phrymaceae, Quillajaceae, Veronicaceae), el reporte de nuevas familias es infrecuente. A nivel de géneros y especies se observan adiciones (e.g. Saldivia et al. 2014, Muñoz-Schick et al. 2016, Calvo \& Moreira-Muñoz 2017), mientras que a nivel de familia esto es muy raro.

Estudios y prospecciones recientes en la región de Arica y Parinacota han permitido mejorar sustantivamente el conocimiento de dicha flora (Trivelli \& Valdivia 2009, Faúndez et al. 2014). Según estos últimos autores, la flora vascular de Arica y Parinacota se compone de alrededor de 600 especies, pertenecientes a 304 géneros y 81 familias.

Entre las plantas vasculares, la más diversa de la región, es la familia Asteraceae, la cual solo recientemente ha recibido tanto un tratamiento detallado (Moreira-Muñoz et al. 2016) como revisiones específicas (Gutiérrez et al.2016). Gracias al trabajo de campo realizado para dicha compilación se ha logrado visitar sitios poco frecuentados de la región. Es el caso de la Quebrada Aroma, al norte del poblado de Socoroma, en la cual se encontró la especie de Anredera que se reporta en este trabajo (Fig. 1).

Durante el trabajo de campo para completar el Catálogo de Asteraceae de la Región de Arica y Parinacota, se encontró una especie de hojas rojas y suculentas. Se trata de la especie Anredera diffusa (Moq.) Sperling. Esta especie se encuentra comúnmente en Perú, pero no había sido registrada en Chile.

\section{Familia Basellaceae}

De acuerdo con Eriksson (2007), esta familia es nativa de las regiones tropicales y subtropicales de América, SE de África y Madagascar. La mayoría de sus especies son trepadoras suculentas que viven en hábitat secos, como matorrales, laderas rocosas, arenas y orillas de caminos, con la excepción de Anredera tucumanensis (Lillo \& Hauman) Sperling y ocasionalmente Tournonia hookeriana Moq., las cuales habitan sitios más húmedos (Eriksson 2007). La familia pertenece al Orden Caryophyllales, cercanamente emparentada con la familia Portulacaceae. Con cuatro géneros y alrededor de 19 especies, la familia muestra una distribución pantropical. Se encuentra posiblemente introducida en Asia (Mabberley 2008), y el mayor número de especies está en América. Los géneros que la componen son Anredera (12 spp., América tropical), Basella (1 sp. pantropical, 1 especie africana y 3 especies de Madagascar); Tournonia (1 sp. de Colombia); y Ullucus (1 sp. andina) (Eriksson 2007).

La familia incluye 2 especies cultivadas como alimento: una, Basella alba L. (espinaca de Malabar o espinaca china), muy usada en la cocina asiática, de origen incierto por lo antiguo de su cultivo, pero probablemente África, y 
la segunda Ullucus tuberosus Caldas, el olluco o ulluku, de origen andino, cultivado en altas altitudes, desde tiempos precolombinos, y que habita desde Venezuela hasta el norte de Argentina. Es hoy un elemento importante de la cocina tradicional de Ecuador y Perú (Eriksson 2009).

Del género Anredera, hay dos especies ampliamente cultivadas como ornamentales: Anredera vesicaria (Lam.) C.F.Gaertn. y Anredera cordifolia (Ten.) Steenis. Esta última, comúnmente llamada "parra de Madeira", es nativa de Bolivia, Argentina, Brasil, Paraguay, Uruguay (Zuloaga et al. 2008). A. cordifolia ha sido recientemente reportada como especie introducida en Valparaíso y la Isla de Pascua (Rodríguez et al. 2108). Ha sido introducida (en muchos casos considerada invasora) en África, Australasia, Europa y Norteamérica. Escapada de cultivo, es bastante agresiva, por la gran producción de tubérculos aéreos.

\section{Anredera Juss. Gen. Pl. 84, 1789.}

Hierbas trepadoras, suculentas, perennes, generalmente con tubérculos; hojas alternas, sésiles o pecioladas, ovadas, cordiformes o elípticas, nervadura poco visible. Inflorescencias en espigas simples o ramificadas, bractéolas
2, en la axila del pedicelo, persistentes o deciduas. Flores pediceladas, perfectas; 5 sépalos persistentes, menores que los 5 pétalos, que son connados en la base; 5 estambres opuestos a los pétalos, filamentos connados y unidos en la base con los pétalos, recurvos en botón, luego erectos, anteras dorsifijas; estilo a veces no diferenciado del estigma, este capitado y luego trilobulado. Fruto globoso, seco, indehiscente, tipo nuez (Eriksson 2009), o utrículo (Martínez-García et al. 1996) generalmente rodeado por el perianto.

Género nativo de los trópicos y subtrópicos de América y el Caribe.

Anredera diffusa (Moq.) Sperling, Monogr. Syst. Bot. Missouri Bot. Gard. 45: 1253. 1993. Basiónimo: Tandonia diffusa Moq., Prod. [A.P.de Candolle] 13(2): 226. 1849.

Planta decumbente, suculenta, de tallos glabros, de color rosado-rojizo (Fig 2A). Hojas principalmente basales, carnosas, con láminas de 2-4 cm largo x 1-2 cmancho, elípticolanceoladas a ovadas o cordadas, cuneadas a subcordadas en la base, con ápice agudo o algo obtuso, angostándose hacia
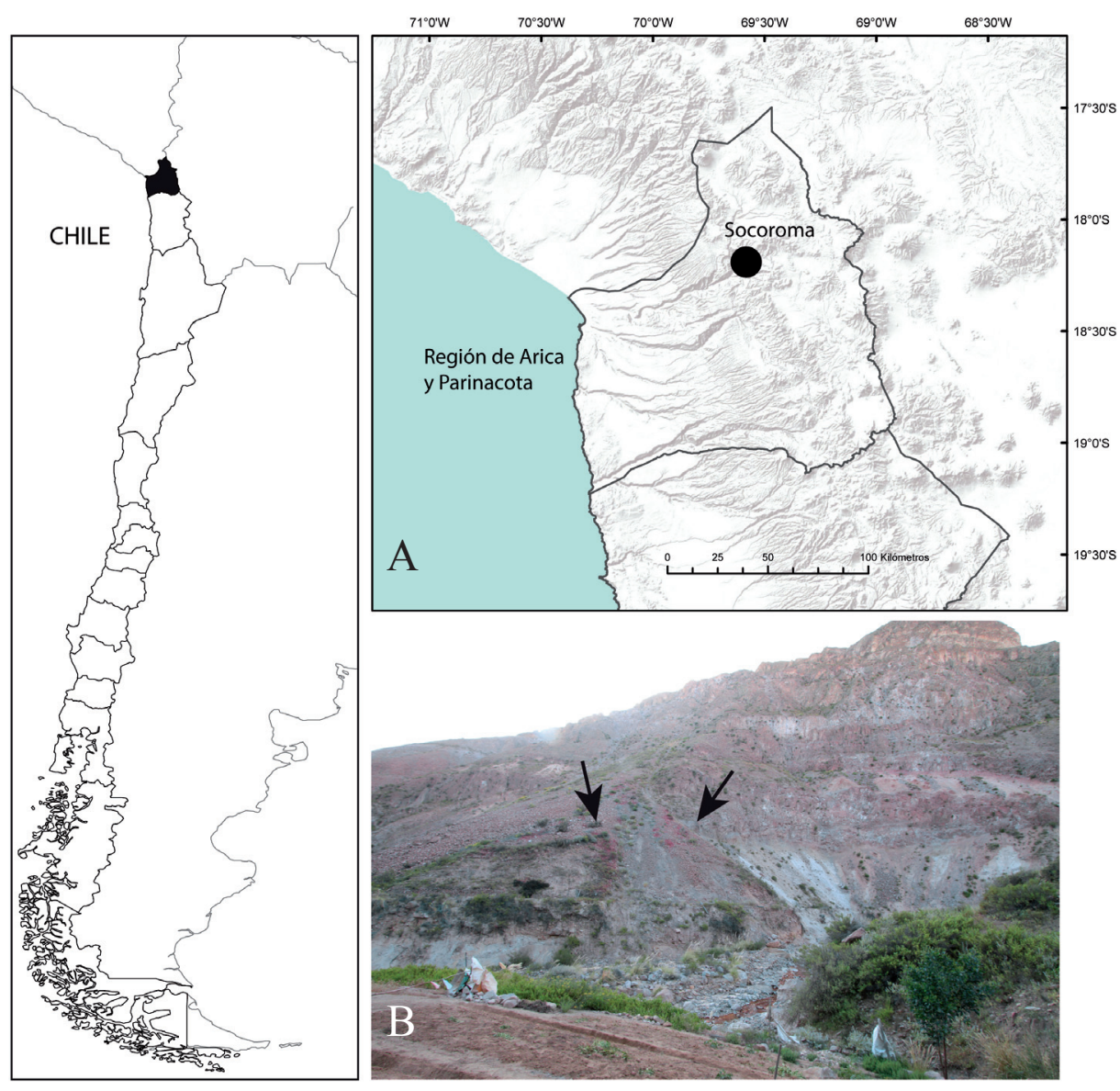

Figura 1. (A) Mapa de ubicación de sitio de estudio. (B) Hábitat de Anredera diffusa en sector de Mancaruma (foto A. Moreira-Muñoz). / (A) Location map of study site. (B) Habitat of Anredera diffusa at Mancaruma locality (photo A. Moreira-Muñoz). 
la base en un pseudopecíolo. Inflorescencias de tallos rojizos de 3-20 cm de largo, sin ramificarse o a veces por sobre las hojas (Fig. 2B). Pedicelos 0,5-1,5 mm de largo, bractéolas persistentes, algo triangulares, de color rosado. Flores de 3 $\mathrm{mm}$ de diámetro, con sépalos ovado-redondeados de $1,1 \mathrm{x}$ $1,1 \mathrm{~mm}$, más cortos que los pétalos, de color rosado intenso en antesis (Fig. 2F), negros y erectos en fruto. Pétalos 1,5 x $1 \mathrm{~mm}$, elípticos a obovados, de color rosado más suave que los sépalos (Fig. 2C, F) pero negros y erectos en fruto. Filamentos angostados hacia las anteras redondeadas, blancas, que emergen de los pétalos; ovario globoso, estilo capitado en antesis, en fruto con un disco carnoso en la base
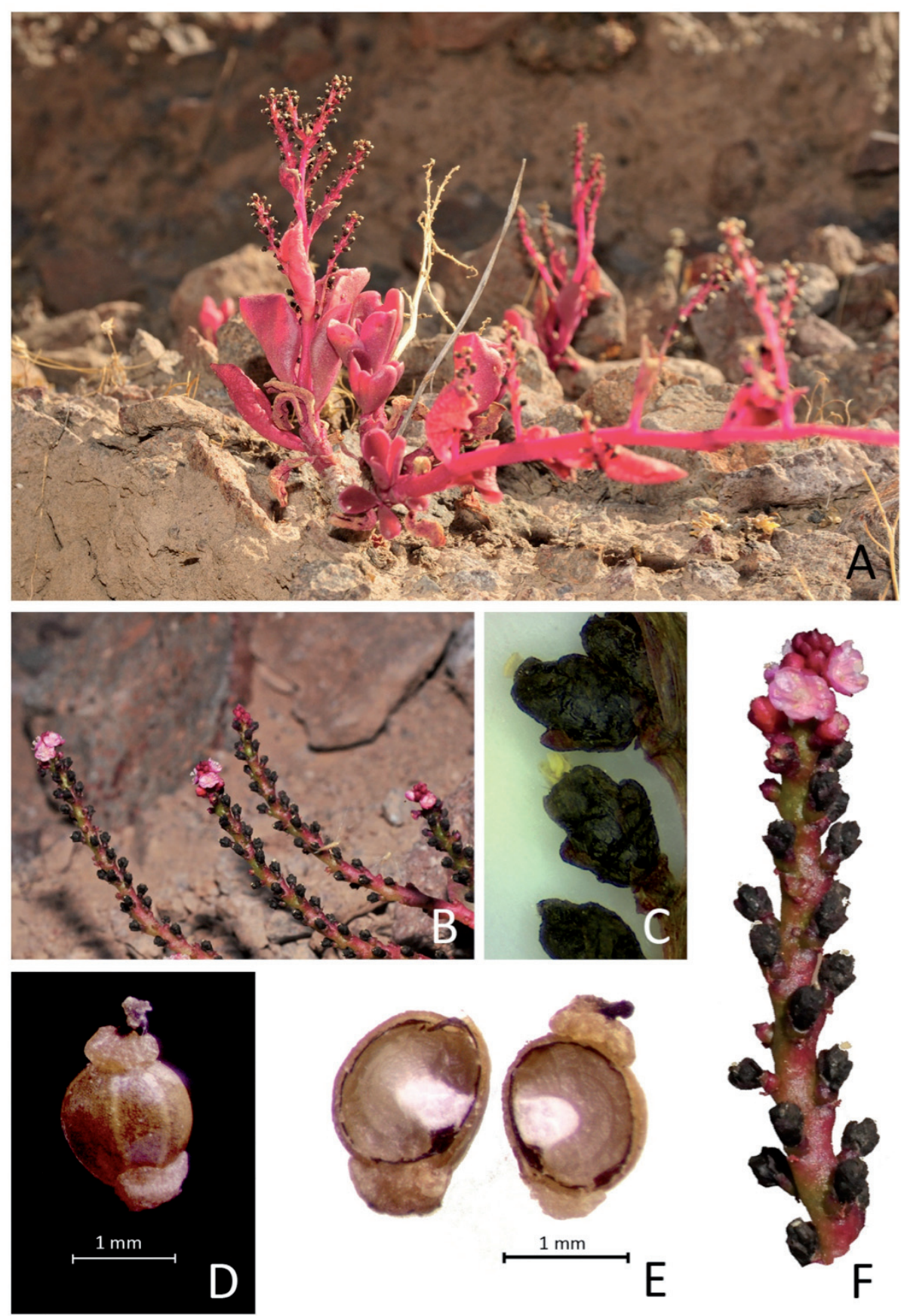

Figura 2. (A) Hábito de la planta. (B) Tallo floral. (C) Flores maduras mostrando estambres exertos. (D) Detalle fruto. (E) Corte de fruto, mostrando la semilla. (F) Detalle tallo floral. Créditos fotos (A,B,F: A. Moreira-Muñoz); (C: foto bajo lupa M. Muñoz); (D,E: fotos bajo lupa N. Smith). / (A) Plant habitus. (B) Floral stem. (C) Mature flowers with exerted stamens. (D) Fruit detail. (E) Fruit cut, showing the seed. (F) Floral stem detail. Photo credits (A, B, F: A. Moreira-Muñoz); (C: M. Muñoz); (D, E: N. Smith). 
y desarrollado en 3 estigmas oscuros muy cortos. Fruto de color café-verdoso brillante, completamente rodeado por sépalos y pétalos ahora negros, de $2 \mathrm{~mm}$ de largo total; contiene una sola semilla (Fig. 2D, E).

La especie fue encontrada en una ladera de difícil acceso, coloreando el paisaje de color rojizo (Fig. 1B). La población ocupa un cono de derrubios, acompañada de especies como: Ambrosia artemisioides Meyen \& Walp. ex Meyen, Solanum lycopersicoides Dunal, Tarasa operculata (Cav.) Kaprov., y Ophryosporus pinifolius (Phil.) R.M. King \& H. Rob.

La especie fue descrita para Perú, donde vive en áreas secas, del centro y sur, tanto en zonas bajas como en altitudes andinas: Lima (Viso, Matucana) y Arequipa. También está indicada para zonas andinas del S de Ecuador. Según Montesinos-Tubée (2013) se desarrolla durante la temporada de lluvias (en época de verano) y florece a partir del mes de marzo. En la zona de Moquegua, Perú, las hojas son utilizadas por sus varias propiedades medicinales. Se separa de otras especies de Anredera principalmente por su perianto de color rosado versus blanquecino de las demás especies (Eriksson 2007).

\section{Material Estudiado}

CHILE, Región de Arica y Parinacota, Quebrada Aroma sector Mancaruma, $18^{\circ} 14^{\prime} 52,4^{\prime \prime} \mathrm{S}-69^{\circ} 36^{\prime} 18^{\prime} \mathrm{W}, 2984 \mathrm{~m}$, 24-V-2016, Moreira 2617 (SGO 168445, CONC); Región de Arica y Parinacota, Quebrada Aroma sector Mancaruma, $18^{\circ} 14^{\prime} 52,7^{\prime}$ ' S-69³6'18” W, 2969 m, 09-VI-2017, Moreira 2791 (SGO 168446).

\section{AGRADECIMIENTOS}

Sebastián Teillier (U. Central) y Daniel Montesinos-Tubée (Perú) aportaron información para la correcta determinación de la especie. El Dr. Roger Eriksson, de la Universidad de Göteborg, Suecia, aclaró dudas y aportó con bibliografía. Natalia Smith y Daniela Escobar, del Jardín Botánico Chagual, tomaron las fotografías bajo lupa. Fondecyt 1180211.

\section{REFERENCIAS}

Calvo, J., Moreira-Muñoz, A. 2017. Chaetanthera peruviana (Asteraceae, Mutisieae), new record for the Flora of Chile. Darwiniana, nueva serie 5(1): 93-97.

Eriksson, R. 2007. A Synopsis of Basellaceae. Kew Bulletin 62: 297-320.

Eriksson, R. 2009. Neotropical Basellaceae. In: Milliken, W., Klitgård, B. \& Baracat, A. (2009 onwards), Neotropikey - Interactive key and information resources for flowering plants of the Neotropics. http://www.kew.org/science/ tropamerica/neotropikey/families/Basellaceae.htm.

Faúndez, L., Flores, R., Faúndez, A., Treumún, M. 2014. Reino Plantae. En: Hernández, J., Estades, C., Faúndez, L., Herreros de Lartundo, J. (eds.) Biodiversidad Terrestre de la Región de Arica y Parinacota, pp. 164-229. Ministerio del Medio Ambiente (MMA), Facultad de Ciencias Forestales y de la Conservación de la Naturaleza, Universidad de Chile, Santiago, Chile.

Gutiérrez, D.G., Muñoz-Schick, M., Grossi, M.A., RodríguezCravero, J.F., Morales, V., Moreira-Muñoz, A. 2016. The genus Stevia (Eupatorieae, Asteraceae) in Chile: a taxonomical and morphological analysis. Phytotaxa 1-18.

MabBerLey, D.J. 2008. Mabberley's Portable Dictionary of Plants, their Classification and Uses. 3rd Edition. Cambridge University Press, Cambridge, 1020 pp.

Marticorena, C. 1990. Contribución a la estadística de la flora vascular de Chile. Gayana Botánica 47(3-4): 85-113.

Martínez-García, J., Avendaño-Reyes, S. 1996. Basellaceae en Flora de Veracruz, fasc. 90: 1-9. Instituto de Ecología, Veracruz, México.

Montesinos-Tubée, D. 2013. Flora de los Andes de Moquehua: Etnobotánica de la cuenca de los ríos Alto Tambo - Ichuña. Cultural Society Inca Legacy Editores, Perú. 300 pp.

Moreira-Muñoz, A. 2011. Plant Geography of Chile. Dordrecht, Springer. 343 pp.

Moreira-Muñoz, A., Muñoz-Schick, M., Marticorena, A., Morales, V. 2016. Catálogo de Asteraceae (Compositae) de la Región de Arica y Parinacota, Chile. Gayana Botánica 73(2): 226-267.

Muñoz-Schick, M., Moreira-Muñoz, A., De Trenqualye, A. 2016. Polycarpon moreiranum M. Muñoz-Schick \& A. de Trenqualye, nueva especie de Caryophyllaceae, endémica de Chile. Gayana Botánica 73(2): 467-470.

Reiche, K. 2013. Geografía Botánica de Chile. Cap. 1, $2^{\text {a }}$ Sección. Estadística General de las Plantas Vasculares Chilenas, págs. 405-413. Obra reeditada por Biblioteca Fundamentos de la Construcción de Chile, Cámara Chilena de la Construcción, Biblioteca Nacional. 556 pp.

Rodríguez, R., Marticorena, C., Alarcón, D., Baeza, C., Cavieres, L., Finot, V.L., Fuentes, N., Kiessling, A., Minoc, M., Pauchard, A., Ruiz, E., Sánchez, P., Marticorena, A. 2018. Catálogo de las plantas vasculares de Chile. Gayana Botánica 75(1): 1-430.

Saldivia, P., Faúndez, L., Marticorena, A., Panero, J.L. 2014. Kieslingia chilensis (Asteraceae: Astereae), a new genus and species from northern Chile. Phytotaxa 177(5): 280290.

Trivelli, M., Valdivia, V. 2009. Alcances sobre Flora y Vegetación de la Cordillera de los Andes: Región de Arica y Parinacota y Región de Tarapacá. 2a edición, SAG, División de Protección de los Recursos Naturales Renovables, Santiago, 180 pp.

Zuloaga, F.O., Morrone, O., Belgrano, M.J. (eds.) 2008. Catálogo de las Plantas Vasculares del Cono Sur (Argentina, Sur de Brasil, Chile, Paraguay y Uruguay). Monographs of the Missouri Botanical Garden 107 (vol. 2 Dicotyledoneae: A-F). 2286 pp.

Recibido: 12.04 .2018

Aceptado: 21.06.2018 